\title{
ULTRASONOGRAPHIC FINDINGS IN PATIENTS EXAMINED IN CATARACT DETECTION-AND- TREATMENT CAMPAIGNS: A RETROSPECTIVE STUDY
}

\author{
Marcio Henrique Mendes, Alberto Jorge Betinjane, Adhele de Sá Cavalcante, \\ Cheng Te Cheng, Newton Kara-José
}

doi: $10.1590 / \mathbf{S 1 8 0 7 - 5 9 3 2 2 0 0 9 0 0 0 7 0 0 0 0 5}$

\begin{abstract}
Mendes MH, Betinjane AJ, de Sá Cavalcante A, Cheng CT, Kara-José N. Ultrasonographic findings in patients examined in cataract detection-and-treatment campaigns: a retrospective study. Clinics. 2009;64(7):637-40.
\end{abstract}

INTRODUCTION: A cataract is defined as an opacity of any portion of the lens, regardless of visual acuity. In some advanced cases of cataracts, in which good fundus visualization is not possible, an ultrasound examination provides better assessment of the posterior segment of the globe.

OBJECTIVES: This study aims to evaluate the ultrasonographic records of patients with advanced cataracts who were examined during cataract campaigns.

METHODS: The ultrasonographic findings obtained from 215 patients examined in cataract campaigns conducted by the Hospital das Clínicas Department of Ophthalmology of the Faculdade de Medicina da Universidade de São Paulo between the years of 2005 and 2007 were evaluated, and the utility of this exam in changing the treatment procedures was studied.

RESULTS: A total of 289 eyes from 215 patients were examined. Of the eyes examined, 77.5\% presented with findings in the vitreous cavity and the posterior pole. A posterior vitreous detachment with no other complications was observed in $47.4 \%$ of the eyes. The remaining $30.1 \%$ presented with eye diseases that could result in a reduced visual function after surgery. The most frequent eye diseases observed were diffuse vitreous opacity (12.1\% of the eyes) and detachment of the retina (9.3\% of the eyes). DISCUSSION: In many cases, the ultrasonographic evaluation of the posterior segment revealed significant anomalies that changed the original treatment plan or contra-indicated surgery. At the very least, the evaluation was useful for patient counseling.

CONCLUSION: The ultrasonographic examination revealed and differentiated between eyes with cataracts and eyes with ocular abnormalities other than cataracts as the cause of poor vision, thereby indicating the importance of its use during ocular evaluation.

KEYWORDS: Ultrasonography; Cataract; Posterior segment; Retinal detachment; Vitreous opacities.

\section{INTRODUCTION}

There are 31 million blind patients in the world and 15 million more with reduced vision. Eighty percent of these are patients with treatable conditions; that is, reversible blindness, and the primary cause is the cataract. ${ }^{1-3}$ A number of factors stands in the way of treatment for cataract prominent among them being emotional factors. ${ }^{4} \mathrm{~A}$ cataract

Ophthalmology Department, Hospital das Clínicas da Faculdade de Medicina da Universidade de São Paulo - São Paulo/SP, Brazil.

Email: marciohmendes@yahoo.com.br

Tel: 55113069.7873

Received for publication on January 27, 2009

Accepted for publication on April 09, 2009 is defined as the opacity of any portion of the lens, regardless of visual acuity. There are various surgical indications, but attempting to re-establish visual acuity to the highest level permitted by the patient's conditions is the most important.

The surgical techniques for the treatment of cataracts have improved substantially over the last few decades due to advances in technology. This has led to more predictable and favorable results with lower costs. A large proportion of the population in developing countries, however, has no access to such procedures. This is due to many factors, including socio-economical and cultural factors, as demonstrated by Alves. ${ }^{5}$ This study reported that $72.86 \%$ of the patients had been suffering from low visual acuity for longer than a year, and $27.14 \%$ for more than 15 years. Of this total, 
$40 \%$ had already had at least one previous indication to phacoemulsification by the National Health Service (SUS), and $80 \%$ had not been submitted for treatment for economic reasons. ${ }^{5}$

The purpose of the cataract campaigns was to make diagnosis and treatment possible in a faster and more accurate way. Nevertheless, it is important to note that during the campaigns, the initial testing and screening may have resulted in a diagnosis of other abnormalities in addition to cataracts. ${ }^{6,7}$ Therefore, the complete semiology of a patient was obtained through a detailed clinical exam. Among the complementary tests used for diagnostic clarification, ultrasound undoubtedly played a decisive role in the course of treatment. Ultrasound is a non-invasive, painless imaging method that can be done at a doctor's practice, even on children. ${ }^{8}$ Ultrasonic evaluation of the posterior pole in patients with opacities is vital to determining the most suitable surgery. ${ }^{9,10}$

The present study aimed to evaluate the records of patients with total cataract from the cataract campaigns of the Hospital das Clínicas da Faculdade de Medicina da Universidade de São Paulo between 2005 and 2007 and to determine the prevalence and nature of intraocular diseases other than cataracts.

\section{MATERIALS AND METHODS}

The results of 289 ultrasonographic ocular exams performed on 215 patients during the cataract campaigns at the Department of Ophthalmology of Hospital das Clínicas da Faculdade de Medicina da Universidade de São Paulo between January, 2005 and January, 2007 were reviewed. All ultrasound examinations had been indicated as a result of the opacity of an eye structure that made the examination of the posterior pole of the ocular globe impossible. All patients were submitted to both A- and B-mode ultrasound at the Hospital das Clínicas da Faculdade de Medicina da Universidade de São Paulo using an Ultra Scan (Alcon, Fort Worth, USA), a B Scan (Quantel Medical, Bozeman, USA) or a Cine Scan (Quantel Medical) using $10 \mathrm{MHz}$ probes. First, patients were evaluated using anamnesis to separate those with a suspected cataract. The patients were then given a complete clinic evaluation, including visual acuity tests, tonometry, biomicrosopy and fundoscopy. In cases in which the evaluation of the fundus of the eye was impossible by the regular procedure due to a high degree of opacity of the lens, the patients were evaluated using ultrasound. When intraocular abnormalities were detected by the ultrasound, the patients were not submitted for cataract surgery.

The ultrasonographic findings of each eye were evaluated to constitute the data used in the present study.
The examinations were conducted with the patient in the horizontal dorsal decubitus position using the transpalpebral technique, utilizing contact gel for ultrasound. Tetracaine ophthalmic drops were placed in the patient's eyes before the test. Examination was systematically performed, evaluating the ocular globe with the ultrasound in longitudinal, axial and transverse cuts of all quadrants. These exams were performed by experienced ultrasonographic doctors of Hospital das Clínicas da Faculdade de Medicina da Universidade de São Paulo. This study was approved by the Institutional Board Review (protocol 0308/07).

\section{RESULTS}

A total of 289 eyes (from 215 patients) were examined. Of this total, $84.7 \%$ presented with total cataract, $10.4 \%$ with corneal opacity, $4.15 \%$ with vitreous opacity and $0.7 \%$ with pupillary seclusion. This data was obtained through biomicroscopic examination using a slit lamp. The age range of the patients was 22 to 97 years old, of which $50.5 \%$ were female and $49.5 \%$ male and $58.1 \%$ were white and $41.8 \%$ were black. Based on the patient anamnesis, $41.5 \%$ of the patients had high blood pressure and $28.7 \%$ had diabetes mellitus. Furthermore, $1.7 \%$ of the patients reported ocular trauma, $0.34 \%$ reported glaucoma and $0.34 \%$ reported congenital cataracts.

The ultrasonographic examinations revealed that $22.5 \%$ of the patients did not present with any abnormalities in the fundus of the eye, $47.4 \%$ presented with posterior vitreous detachment, $9.3 \%$ presented with retinal detachment and $12.1 \%$ presented with diffuse vitreous opacity. Signs of introflexion of the sclera were observed in $0.7 \%$ of the patients. In $4.5 \%$ of the patients, an enlargement of the

Table 1- Percentage of patients examined in the cataract campaigns in which abnormalities of the eye were detected.

\begin{tabular}{lc}
\hline Diagnosis & Percentage \\
\hline Posterior vitreous detachment & $47.4 \%$ \\
Vitreous opacities & $12.1 \%$ \\
Retina detachment & $9.3 \%$ \\
Introflexion of the sclera & $0.7 \%$ \\
Enlargement of the optic nerve & $4.5 \%$ \\
excavation & \\
Papillary druses & $0.7 \%$ \\
Staphylomas & $0.7 \%$ \\
Retinoschisis & $0.7 \%$ \\
Vitreoschisis & $0.35 \%$ \\
Silicon oil in the vitreous cavity & $0.35 \%$ \\
Without changes & $22.5 \%$ \\
\hline
\end{tabular}


optic disc excavation was observed, $0.7 \%$ showed signs of papillary druses, $0.7 \%$ showed staphylomas, $0.35 \%$ showed retinoschisis, $0.35 \%$ showed vitreoschisis, $0.35 \%$ showed sinquisis scintilans and $0.35 \%$ presented with signs of silicon oil in the vitreous cavity.

\section{DISCUSSION AND CONCLUSION}

Ultrasonographic evaluation is often used in cataract campaigns performed by the Hospital das Clínicas da Faculdade de Medicina da Universidade de São Paulo and is particularly indicated in cases in which the posterior pole cannot be examined through normal optic methods.

It is important to note that in cases involving lens opacity, the potential acuity meter (PAM) can be used to estimate post-operative visual acuity. The PAM is an important test that should be applied prior to cataract surgery, especially in eyes in which the evaluation of the ocular fundus (mainly the macular) is impossible by regular optic methods. This ocular test, however, is only useful to evaluate the potential acuity when there are no significant opacities of the lens. Because of this fact, the PAM is not usually used in cataract campaigns.

The results of the present study demonstrated that out of the 289 eyes with medium to profound opacity of the lens, $77.5 \%$ presented with vitreous detachment, which can be a natural occurrence during senescence. ${ }^{11}$ In $30.1 \%$ of the eyes, the ultrasound revealed eye abnormalities that could compromise the function of the eye after surgery. The most common of these abnormalities was vitreous opacities $(12.1 \%)$, followed by retinal detachment $(9.3 \%)$. The patients with these anomalies were not considered for cataract surgery and were guided to adequate treatment for the detected disease.

Some of our findings do not agree with the previous literature, particularly studies related to prevalence of the various abnormalities. According to Corrêa et al., posterior vitreous detachment was most commonly found using ultrasound $(26.1 \%)$, followed by retina detachment $(9.7 \%)$ and vitreous hemorrhage $(8.6 \%) .{ }^{12}$ Similarly, Anteby et al. reported that posterior staphyloma was more frequent $(7.2 \%) .{ }^{13}$ These studies, however, did not examine patients evaluated during cataract campaigns. Moreover, the subjects/patients in the previous studies were divided into two groups, those with and those without a history of ocular trauma. Significant differences were detected in the diagnosis of these two groups. The fact that the exams were not always conducted by the same physician does not affect the outcome of the exam, as all physicians were well trained in conducting ultrasonographic examinations. Furthermore, when there was doubt in the diagnosis, another physician was always consulted for clarification.

In conclusion, despite statistical inconsistencies regarding the prevalence of diseases of the posterior segment, our study further demonstrates that ultrasonographic evaluation is an important pre-surgical step in patients with dense cataracts or other opacities. The results of this study allow us to highlight the importance of ultrasonographic evaluation in cataract campaigns. We found that during the cataract campaigns, it was possible to detect and diagnose severe ocular diseases, making it possible for the patient to treated properly. Most importantly, the ultrasound also supplied useful information for determining if lens surgery should be performed or if alternative treatments are recommended.

\section{REFERENCES}

1. Thylefors B, Négrel AD, Pararajasegaram R, Dadzie KY. Global data on blindness. Bull World Health Organ. 1995;73:115-21.

2. Resnikoff S, Pararajasegaram R. Blindness prevention programmes: past, present and future. Bull World Health Organ. 2001;79:222-6.

3. Thylefors B, Resnikoff S. Progress in the control of world blindness and future perspectives. Santé. 1998;8:140-3.

4. Marback R, Temporini E, Kara Júnior N. Emotional factors prior to cataract surgery. Clinics. 2007;62:433-8.

5. Alves MR, José NK, da Silva AL, Prado Júnior J, Temporini ER. Senile cataract: patient's characteristics and perceptions on a sight restoration communitary project. Rev Hosp Clin Fac Med Sao Paulo. 1997;52:16-9.
6. Saad Filho R, Saad FGL, Freitas LL. Cost of phacoemulsification in the national campaign of elective cataract surgery in Itápolis, SP, Brazil. Arq Bras Oftalmol. 2005;68:55-9.

7. Temporini ER, Kara-Jose N. Visual loss: prevention strategies. Arq Bras Oftalmol. 2004;67:597-601.

8. Walsh A. Métodos e técnicas de exame. In: Abreu G, editor. Ultrasonografia ocular: atlas e texto . $3^{\mathrm{a}}$ ed. Rio de Janeiro: Cultura Médica; 2002. P.13-8.

9. Nassaralla Jr JJ, Nassaralla BA. The role of ecography before catarct surgery. Rev Bras Oftalmol. 2003;62:480-4. 
10. Byrne SF, Green RL. Orbital Tumors. In: Byme SF, Green RL, editors. Ultrassound of the eye and orbit. St. Louis: Mosby Year Book; 1992. p.53-8.

11. Rosen E. Patient workup for cataract surgery. In: Yanoff M, Duker JS, editors. Ophthalmology. London: Mosby International; 1999. p.181-4.
12. Corrêa ZMS, Goldhardt R, Marcon AS, Marcon IM. Ultrasound findings in patients with dense cataracts. Arq Bras Oftalmol. 2002;65:609-13.

13. Anteby II, Blumenthal EZ, Zamir E, Waindin P. The role of preoperative ultrasonography for patients with dense cataract: a retrospective study of 509 cases. Ophthalmic Surg Laser. 1998;29:114-8. 